\title{
FORMACIÓN EN COMPETENCIAS DIGITALES PARA ESTUDIANTES UNIVERSITARIOS BASADA EN EL MODELO DIGCOMP
}

\section{DIGITAL COMPETENCES TRAINING FOR UNIVERSITY STUDENTS BASED ON DIGCOMP MODEL}

\author{
Víctor González Calatayud; victor.gonzalez@um.es \\ Marimar Román García; mariamar.roman@um.es \\ María Paz Prendes Espinosa; pazprend@um.es \\ Universidad de Murcia
}

\section{RESUMEN}

La competencia digital es una de las competencias básicas del ciudadano del siglo XXI y es además una de las competencias transversales de todas las universidades españolas. En este contexto, cobra especial relevancia la formación en competencias digitales de los estudiantes universitarios y para ello hemos llevado a cabo la experiencia que describimos en este artículo. Hemos diseñado una propuesta formativa basada en un método de aprendizaje por tareas y sustentada en las 5 dimensiones de la competencia digital de la Comisión Europea. Hemos recogido datos de evaluación con un diseño pretest-postest aplicando un cuestionario. Tras evaluar la experiencia, nuestros estudiantes han expresado su satisfacción con la metodología y han puesto en evidencia la adquisición de la competencia.

Palabras clave: competencia digital, formación, estudiantes, universidad

\begin{abstract}
Digital competence is one of the basic competences of citizenship in the 21st century and is also one of the general competences of all Spanish universities. In this context, the training of university students in digital competence is particularly important and we have carried out the experience described in this article. Our instructional design has been designed based on a task-based learning method and it is based on European Comission's proposals with 5 dimensions. The evaluation was done with a pretestpostest using a questionnaire. After evaluating the experience, our students have expressed their satisfaction with the methodology and have highlighted the acquisition of competence.
\end{abstract}

Keywords: digital competence, training, students, university 


\section{FORMACIÓN EN COMPETENCIAS DIGITALES.}

La competencia digital es una de las competencias básicas de los ciudadanos del siglo XXI y además está incluida como competencia transversal en todas las universidades españolas a partir de la llegada de la Convergencia Europea. Por otra parte, la innovación educativa en nuestro sistema de enseñanza superior ha de convertirse en un elemento básico de nuestro quehacer diario si queremos adaptar nuestras instituciones al contexto laboral y económico de nuestra sociedad actual. La "Agenda Digital para Europa" de la Comisión Europea (2014) nos explica que "la economía digital crece siete veces más deprisa que el resto de la economía" y se prevé que en 2020 tendremos "16 millones más de puestos de trabajo que requieran competencias en tecnologías de la información y la comunicación" (p. 3). Y es en este contexto donde cobra sentido formar en competencias digitales a todos nuestros estudiantes.

\subsection{La competencia digital}

De los muchos conceptos que se han elaborado sobre la competencia, a los efectos de nuestro trabajo nos quedamos con el de Pavié (2011, p. 77) quien entiende que "es un grupo de elementos combinados (conocimientos, destrezas, habilidades y capacidades) que se movilizan e integran en virtud de una serie de atributos personales, en contextos concretos de acción". Este concepto es coincidente con la explicación más sencilla de Monereo (2005, p. 13) quien considera que "alguien competente es una persona que sabe leer con exactitud qué tipo de problema es el que se le plantea y cuáles son las estrategias que deberá activar para resolverlo".

A partir de la importancia de las competencias como elemento central de un renovador enfoque de nuestros sistemas de enseñanza, se comienzan a elaborar -por parte de instituciones y organismos oficiales- listados de competencias básicas y en todos ellos se incluye la competencia digital (OCDE, 2005; Comisión Europea, 2006; LOE, 2006; Comisión Europea, 2013; UNESCO, 2011; LOMCE, 2014; Comisión Europea, 2016; INTEF, 2017).

En los trabajos sobre competencia digital se parte de la idea de que es una competencia necesaria en la formación del ciudadano del siglo XXI, pues es necesaria además para afrontar el aprendizaje a lo largo de la vida. Sobre este tema es necesario citar el trabajo pionero realizado en el marco del proyecto DeSeCo (OCDE, 2005), en el cual se determinan las competencias clave para el ciudadano del siglo XXI: usar las herramientas de forma interactiva (lenguaje, símbolos, conocimiento, información y tecnologías en general); interactuar con grupos heterogéneos (relacionarse, cooperar, trabajar en equipo y resolver conflictos); y tercero, actuar de manera autónoma (en cualquier contexto, conducir planes de vida y defender derechos e intereses). En la propuesta reelaborada (OCDE, 2009) se definen las tres dimensiones como información, comunicación e impacto ético-social.

En la definición de la Comisión Europea (2006, p. 15) la competencia digital "entraña el uso seguro y crítico de las tecnologías de la sociedad de la información (TSI) para el trabajo, el ocio y la comunicación". De forma general, las definiciones de competencia digital pueden clasificarse en relación a dos grandes perspectivas: por un lado, las que enfatizan el componente tecnológico y, por otro lado, las que hacen 
hincapié en la dimensión informacional o comunicativa (Gutiérrez, 2014). Esta autora, englobando ambas tendencias, considera que la competencia digital se ha de entender como "valores, creencias, conocimientos, capacidades y actitudes para utilizar adecuadamente las tecnologías, incluyendo tanto los ordenadores como los diferentes programas e Internet, que permiten y posibilitan la búsqueda, el acceso, la organización y la utilización de la información con el fin de construir conocimiento" (Gutiérrez, 2014, p. 54).

El modelo más conocido de competencia digital es el DigComp (Comisión Europea, 2013; Ferrari, Neza y Punie, 2014; Comisión Europea, 2016). En este modelo se parte de un concepto globalizador de competencia -que incluye conocimientos, habilidades y actitudes- y se incluyen como áreas de la competencia digital las siguientes: información, comunicación, creación de contenidos, seguridad y solución de problemas.

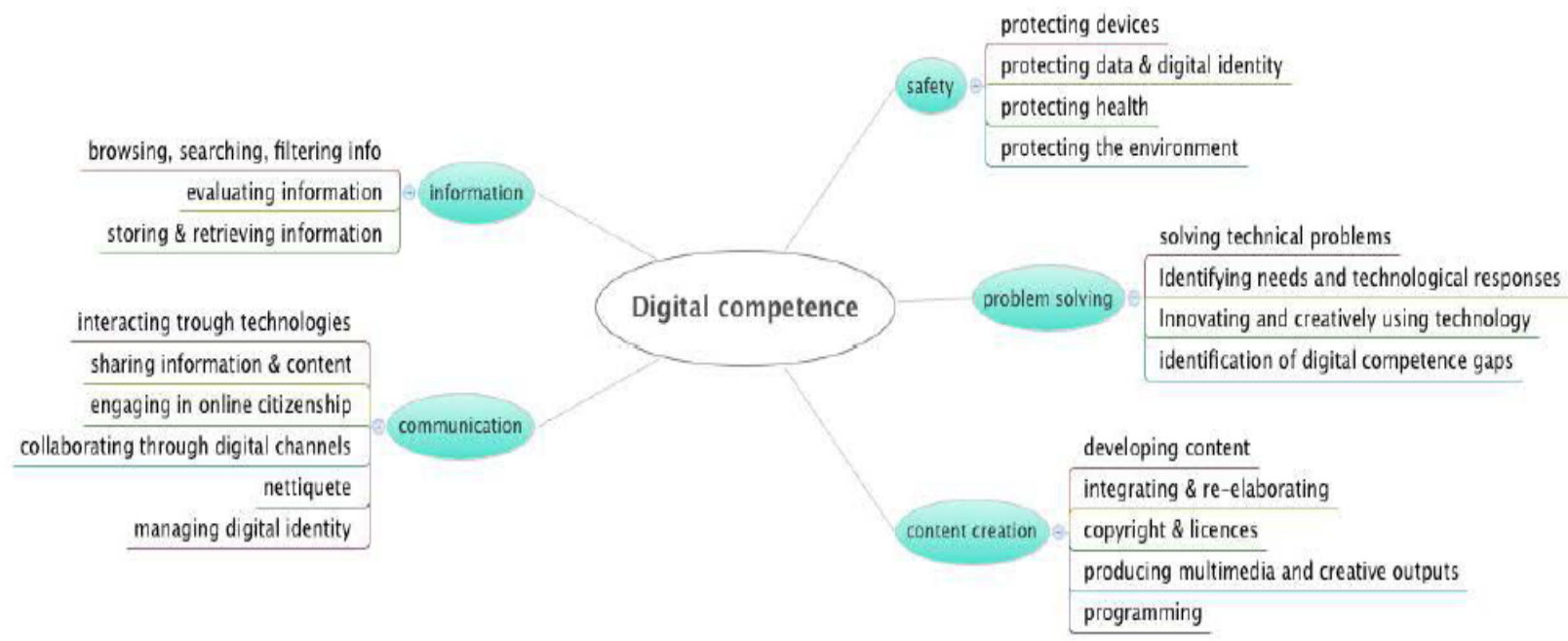

Figura 1: Áreas y competencias del Modelo DigComp (Ferreri, Neza y Punie, 2014, p. 9)

Este modelo es posteriormente revisado y completado por trabajos posteriores (Carretero, Vuorikari y Punie, 2017; Marín, 2017), pero en cualquier caso se siguen considerando en todos ellos esas cinco dimensiones básicas. Por ello, esas han sido las cinco dimensiones en las que hemos sustentado nuestra experiencia de trabajo con los estudiantes universitarios que presentamos a continuación.

Dentro del marco de la competencia digital podemos distinguir distintos tipos, para los que se ha adaptado el modelo de forma más concreta, además de una gran cantidad de trabajos relacionados como recoge Petterson (2017). De este modo, la competencia digital docente se encuentra como un aspecto clave dentro de la educación. Recientemente el INTEF ha elaborado un Marco Común donde se recogen los aspectos esenciales de la misma (INTEF, 2017). También podemos encontrar el Marco Europeo para Organizaciones Educativas Digitalmente Competentes, el cual se considera como aspecto esencial para mejorar la competencia digital de toda la comunidad educativa (Kampylis, Punie y Devine, 2016). Y, por supuesto, la 
competencia digital del alumnado, aspecto central de este trabajo y que en el siguiente apartado se analiza.

\subsection{La competencia digital de los estudiantes universitarios}

En el año 2001, Prensky diferenció entre "nativos digitales" (aquellos que habían nacido en la era de las TIC) e "inmigrantes digitales" (los que habían nacido anteriormente a la existencia de las redes y por tanto requieren de un periodo de aprendizaje y adaptación siendo ya adultos). Añadidos a los conceptos anteriores, encontramos otros como Baby Boomers, la Generación X, Y o Z-Generación y Generación Net (Dávila, 2006). En este sentido encontramos también la teoría de los "visitantes" y los "residentes" propuesta por White y Le Cornu (2010). No obstante, en una investigación reciente (Prendes y Román, 2017), los datos recogidos con más de 2000 estudiantes universitarios, mostraron que en España no siempre nuestros estudiantes son tan hábiles como podría parecer con el uso de las tecnologías como herramientas para el aprendizaje en su actividad académica.

Area (2010) justifica la necesidad de formar en competencias digitales al alumnado universitario porque deben ser capaces de acceder a la nueva información, formarse en habilidades de búsqueda, desarrollar su capacidad de construir conocimiento en interacción, saber expresarse y comunicarse con los nuevos lenguajes y herramientas $\mathrm{y}$, por último, adquirir competencias para el aprendizaje en espacios enriquecidos y construidos con tecnologías.

A partir de estos planteamientos y de los desarrollos de la competencia digital y la ciudadanía digital, surgen iniciativas centradas en la evaluación de las capacidades, habilidades y actitudes de los alumnos frente a las posibilidades de las herramientas digitales. Con esta finalidad, encontramos el proyecto Ikanos sobre competencias digitales que, de modo general, presenta un instrumento de autoevaluación de la competencia digital basado en el modelo DigComp.

De modo específico y centrando sus propuestas sobre la competencia digital del alumnado universitario, encontramos los trabajos del grupo de la Universidad de Alicante dirigido por Roig. En Roig y cols. (2012) se presenta un cuestionario de "Competencias básicas digitales 2.0 de los estudiantes universitarios" (COBADI) que incluye uso general de Internet, uso de TIC para la comunicación y la colaboración, uso de TIC para búsqueda y tratamiento de la información, las competencias interpersonales para usar TIC en la universidad y un último bloque sobre herramientas virtuales y de comunicación social en la universidad. Roig y Pascual (2012) utilizan el trabajo previo de Guzmán (2008) y analizan la competencia digital del alumnado de magisterio incluyendo las áreas de uso del ordenador, uso y frecuencia de uso de las TIC, y por último competencias básicas de uso de TIC. En Flores y Roig (2016) se utiliza un cuestionario que se aplica a alumnado de Pedagogía e incluye el ámbito pedagógico-social, los aspectos éticos y legales, la dimensión técnica, la gestión escolar y el desarrollo profesional.

En Cabezas, Casillas y Pinto (2014) se realiza un análisis de la autopercepción de la competencia digital con alumnado de Grado de Educación Primaria y un instrumento diseñado ad hoc con cinco bloques: conocimientos básicos de la web 2.0, información, 
uso de diferentes tecnologías, uso de servicios de la web 2.0 y por último, uso de TIC en educación.

El cuestionario INCOTIC (Inventario de Competencias TIC) es también una herramienta elaborada específicamente para la autoevaluación de la competencia digital del alumnado universitario (Gisbert, Espuny y González, 2011). Este cuestionario tiene 6 bloques: datos sociodemográficos; acceso a los recursos digitales; grado de uso de las TIC en general; formación en TIC o por medio de TIC; autopercepción de su competencia TIC, entendida como alfabetización tecnológica, uso de TIC como recursos de aprendizaje, incidencia de TIC en tanto que ciudadanos competentes, uso de TIC para el trabajo intelectual, como herramienta de información y como herramienta de comunicación; y por último, una sección sobre su actitud frente a las TIC, planteando diversas situaciones y contextos de aplicación para que ellos puedan expresar su opinión.

Por último, hemos de citar el ejemplo modélico del sistema educativo donde más se trabaja la competencia digital, que es el escandinavo según Ferrari, Neza y Punie (2014), sistema que ha sido bien descrito y analizado por Krumsvik (2008).

\section{METODOLOGÍA}

Para la realización de este proyecto se utilizó un diseño cuasi-experimental en el que se ha intentado ver la diferencia existente en un grupo después de trabajar las distintas áreas competenciales del modelo DigComp ya citado. Para ello se ha realizado un diseño de pretest-postest y una serie de actividades diseñas explícitamente para trabajar cada una de las áreas.

\subsection{Objetivos}

El propósito principal de este proyecto fue mejorar la competencia digital del alumnado de $2^{\circ}$ de pedagogía mediante la realización de tareas enfocadas a trabajar cada una de las áreas competenciales del Marco Europeo. Más concretamente, se propusieron los siguientes objetivos: (1) Conocer la competencia digital del alumnado antes de comenzar la asignatura; (2) Trabajar la competencia digital mediante la realización de tareas grupales; y (3) determinar el nivel de mejora de la competencia digital del alumnado después de la finalización del proyecto.

\subsection{Participantes}

La muestra recogida fue por conveniencia, ya que los participantes en el proyecto fueron los matriculados en la asignatura "TIC para la formación" del Grado en Pedagogía. Al finalizar se recogieron datos de un total de 115 alumnos. De estos, el $81.7 \%$ fueron mujeres y la media de edad fue de 21.37 (DT $=2.65$ ), con edades comprendidas entre los 19 y 33 años. Igualmente, el $78.3 \%$ era la primera vez que se matriculaban en la asignatura. El 25.2\% indicó tener formación en relación con las Tecnologías digitales.

En cuanto al equipamiento del que disponían destaca que el 43.5\% disponía tanto de un ordenador, ya fuera fijo o portátil, y de un teléfono inteligente con conexión a Internet. A lo cual se añade que el $28.7 \%$ tenía, además de lo anterior, una Tablet. 


\subsection{Instrumento}

Para analizar la competencia digital del alumnado se ha utilizado el cuestionario de autodiagnóstico elaborado en el proyecto lkanos (se puede encontrar aquí) impulsado por el Gobierno Vasco y basado en el Marco Europeo de la Competencia Digital (Comisión Europea, 2006 y 2016). Las áreas de competencia (Figura 1) se estructuran en torno a 5 ejes principales, sobre las cuales se estructuran las 21 competencias definidas. El cuestionario mostró una gran fiabilidad para los datos, siendo el alfa de Cronbach de .943 en el pretest y de .953 en el postest para todo el cuestionario. Igualmente, las dimensiones de las que está compuesto también obtuvieron un buen resultado siendo en todos los casos superior a .70: Información, $\alpha=.84$; Comunicación, $\alpha=.73$; Creación de contenidos, $\alpha=.90$; Seguridad, $\alpha=.76 ;$ y Resolución de problemas, $\alpha=.83$.

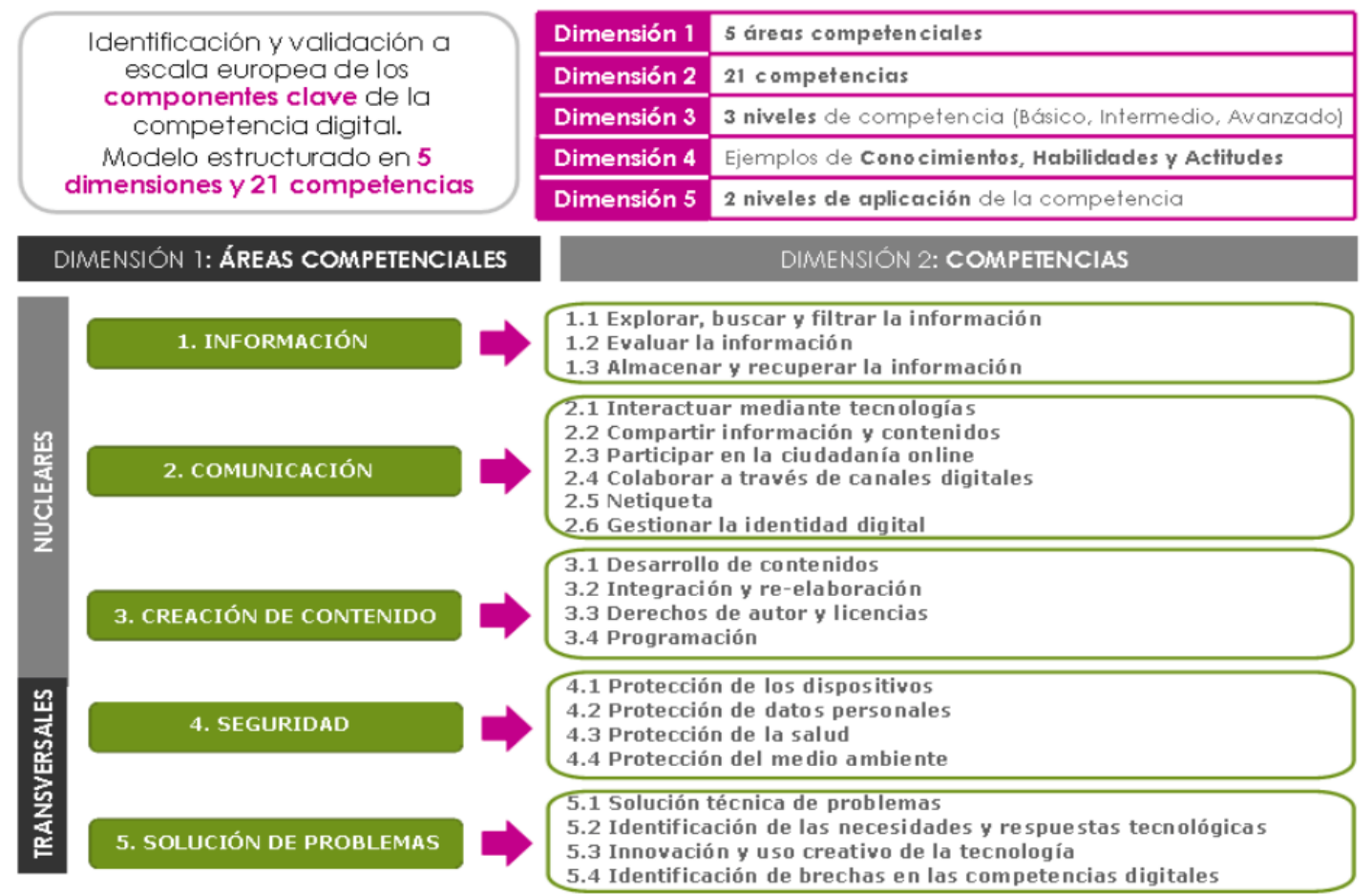

Figura 2. Modelo DIGCOM, cuadro resumen elaborado dentro del Proyecto Ikanos del Gobierno Vasco. Fuente: http://www.innova.euskadi.eus/v62-ikanos2/es/

\subsection{Actividades realizadas para trabajar la Competencia Digital}

Tras realizar el pretest y a lo largo del cuatrimestre, el alumnado realizó 5 tareas con una duración de dos semanas cada una. Cada tarea se vinculó a una de las dimensiones de la competencia digital en el marco de DigComp:

$1^{a}$ tarea: curación de contenidos. Área: información.

La finalidad de esta tarea era la curación de contenidos y para ello cada uno de los grupos debía elegir un tema diferente en el cual profundizar, todos ellos relacionados con la asignatura. Posteriormente dentro de cada grupo cada alumno debía hacer una búsqueda de información diferente en relación con el tema escogido: (1) 
Investigaciones y artículos científicos, (2) noticias actuales en prensa, (3) información en redes sociales, (4) opiniones personales y (5) imágenes y vídeos. Una vez finalizada la búsqueda en grupo, seleccionaron la información más relevante sobre el tema y elaboraron un artículo breve que debían compartir en abierto a través de la herramienta Scoop.it.

$2^{\circ}$ tarea: uso seguro de internet. Área: seguridad.

La finalidad de esta tarea era el diseño y puesta en marcha de una dinámica de grupo cuyo objetivo debía ser trabajar algunos de los riesgos más comunes asociados al uso de internet. Las instrucciones de la dinámica se recogerían en un póster digital y debían incluir los siguientes puntos: Introducción, objetivos, destinatarios, duración, materiales necesarios para llevarla a cabo, desarrollo, autores y, por último, consejos y sugerencias. Cada una de las dinámicas se llevó a cabo en clase.

$3^{\circ}$ tarea: elaboración de infografías. Área: creación de contenidos

Esta actividad consistía en la creación de una infografía a partir de la lectura de artículos académicos dentro del campo de la Tecnología Educativa. Para ello los alumnos eligieron uno de los artículos de la Revista Interuniversitaria de Investigación en Tecnología Educativa (RIITE) para posteriormente seleccionar la información relevante del artículo elegido y transformarla en una infografía, utilizando para ello alguna de las herramientas gratuitas de las que disponen en la red y que ellos debían elegir.

\section{$4^{\circ}$ tarea: plan de comunicación en red. Área: comunicación}

Esta tarea estaba directamente relacionada con la tarea anterior, ya que el alumnado diseñó un plan de comunicación en red cuyo objetivo era difundir la infografía realizada, plan que, entre otras estrategias, debía incluir una vídeo-reseña de elaboración propia. Una vez diseñado el plan de difusión en red (concretando la audiencia, objetivos claros y realistas, canales de comunión a emplear, estrategias, herramientas y temporalización), pasaban a la elaboración de la vídeo-reseña del artículo. Finalmente debían aportar evidencias de la difusión realizada.

\section{$5^{\circ}$ tarea: casos prácticos. Área: resolución de problemas}

En esta tarea el alumnado tenía que resolver 3 de los 6 casos prácticos que se les planteaban (a elegir por ellos). Una vez seleccionados los tres casos a resolver, se trataba de dar las respuestas a cada uno de los casos revisando los contenidos aprendidos en la asignatura y enfocando las posibles soluciones desde su perfil profesional. Todos los casos planteados estaban relacionados con la resolución de problemas técnicos, la identificación de necesidades y respuestas tecnológicas, la innovación y uso creativo de las tecnologías y también la identificación de brechas relacionadas con las competencias digitales.

Estas 5 tareas se reflejaban en el blog de cada uno de los grupos con las evidencias de cada uno de los pasos dados para el correcto desarrollo de las mismas. 


\section{RESULTADOS}

\subsection{Datos previos a la realización: Pretest}

En el área de Información la media antes de comenzar con todo el proceso de trabajo fue de $78.98(D T=15.43)$ con un rango que iba desde 47 a 108 . Esta media fue superior en varones $(M=88.5, D T=14.85)$ que en mujeres $(M=77.5, D T=15.07)$, siendo significativa la diferencia, $t(113)=2.55, p=.012$. En cuanto a la Comunicación, los datos muestran una media de $87.05(D T=14.44)$ en un rango entre 57 y 126 . En esta área no se encontraron diferencias significativas entre hombres $(M=90.93, D T=$ 17.2) y mujeres $(M=86.44, D T=13.98), t(113)=1.08, p=.28$. En la Creación de contenidos, los alumnos participantes obtienen una media de 79.97 ( $D T=25.67$ ), con un mínimo de 20 y un máximo de 135. En esta área competencial, aunque la media de los varones $(M=93.5, D T=34.52)$ es superior a la de las mujeres $(M=77.87, D T=$ 23.56), las diferencias no fueron significativas, $t(14.94)=1.63, p=.12$.

En el área transversal de Seguridad la media obtenida fue de 88.98 ( $D T=17.08)$, con un mínimo de 38 y un máximo de 128. Se encontraron diferencias significativas entre mujeres $(M=87.57, D T=16.64)$ y hombres $(M=98.07, D T=17.71), t(113)=-2.18, p$ $=.032$. Por último, en el área transversal de Resolución de problema la media fue de $66.26(D T=15.38)$, siendo el mínimo obtenido 32 y el máximo 102. Al igual que en la otra área transversal, existieron diferencias significativas entre mujeres $(M=64.97, D T$ $=14.91)$ y varones $(M=74.57, D T=16.29), t(113)=-2.21, p=.029$. En la siguiente Figura 3 se puede observar las diferencias en varones y mujeres. Las puntuaciones se muestran en relación al total en bruto del instrumento.

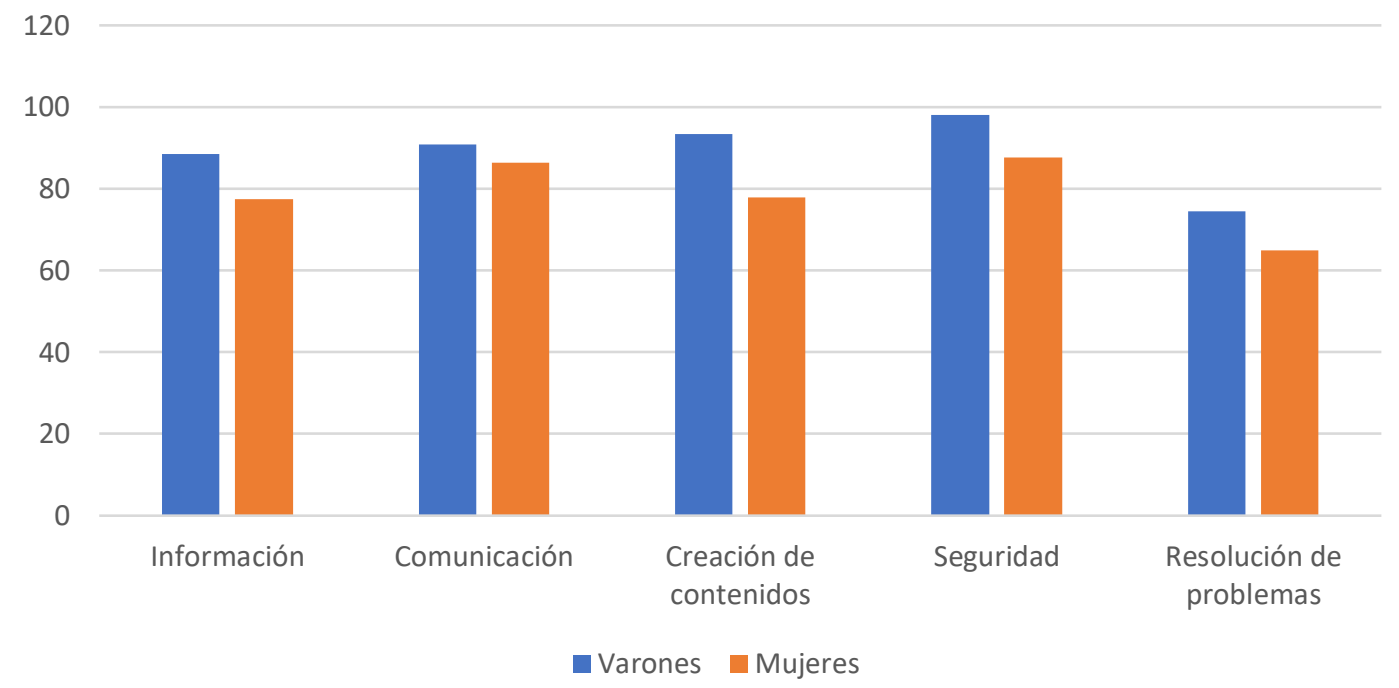

Figura 3 Diferencia de medias entre varones y mujeres en las 5 áreas competenciales en el pretest

\subsection{Datos después de trabajar la competencia digital: Postest}

Después de trabajar la competencia digital la media en el área competencial de Información fue de $86.17(D T=16.01)$, con un máximo de 119 y un mínimo de 44. En este caso la media de las mujeres $(M=85.79, D T=16.29)$ se ha igualado al de los varones $(M=87.86, D T=14.91)$, dejando de ser la diferencia significativa, $t(113)=$ $0.53, p=.59$. En el área competencial de Comunicación también ha subido la media, 
siendo en esta ocasión de $95.84(D T=14.96)$, con valores que van desde los 56 hasta los 136. Al igual que sucedía en el pretest, no se encontraron diferencias significativas entre mujeres $(M=94.81, D T=15.26)$ y los varones $(M=100.48, D T=12.81), t(113)$ $=-1.58, p=.117$. En el área de Creación de contenidos es donde más sube la media con respecto al pretest, teniendo un valor de $100.01(D T=20.89)$, en valores que van desde los 32 a los 150. La media de los varones $(M=106.71, D T=22.42)$ fue superior al de las mujeres $(M=98.51, D T=20.36)$, pero no significativa, $t(113)=-1.64, p=$ .104.

En las áreas competenciales transversales también aumenta la media. En el caso del área de Seguridad la media fue de $98.81(D T=17.44)$. La media de los varones $(M=$ $103.38, D T=13.14)$ fue algo superior del de mujeres $(M=97.79, D T=18.64)$ pero no fue significativa la diferencia, $t(113)=1.33, p=.185$. Por último, en el área de Resolución de problemas la media fue de 73.63 (DT =13.56), con un mínimo de 15 y un máximo de 101. Se hallaron diferencias significativas en la media entre mujeres $(M$ $=72.4, D T=13.99)$ y la de varones $(M=79.14, D T=9.95), t(113)=-2.09, p=.039$.

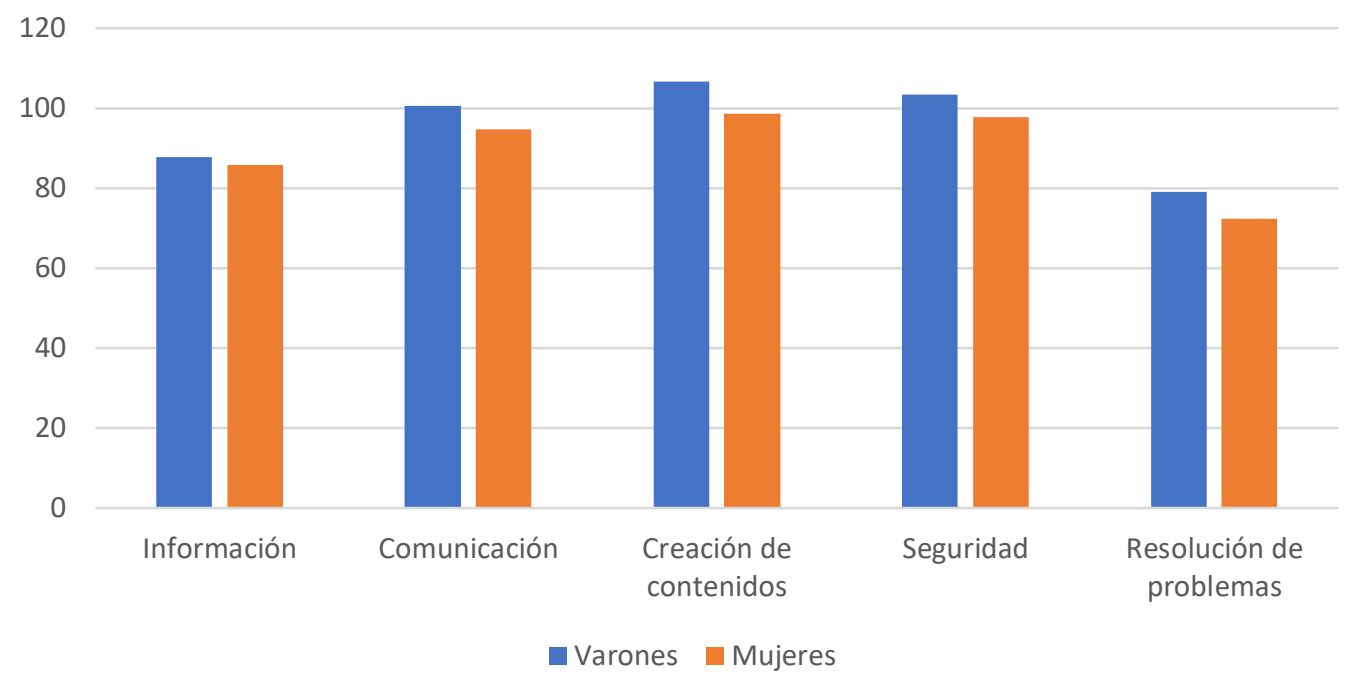

Figura 4 Diferencia de medias entre varones y mujeres en las 5 áreas competenciales en el postest

\subsection{Comparativo pretest - postest}

Como se observa en la Figura 2, los cambios en el pretest-postest en todas las áreas de la competencia digital de los alumnos es evidente. 


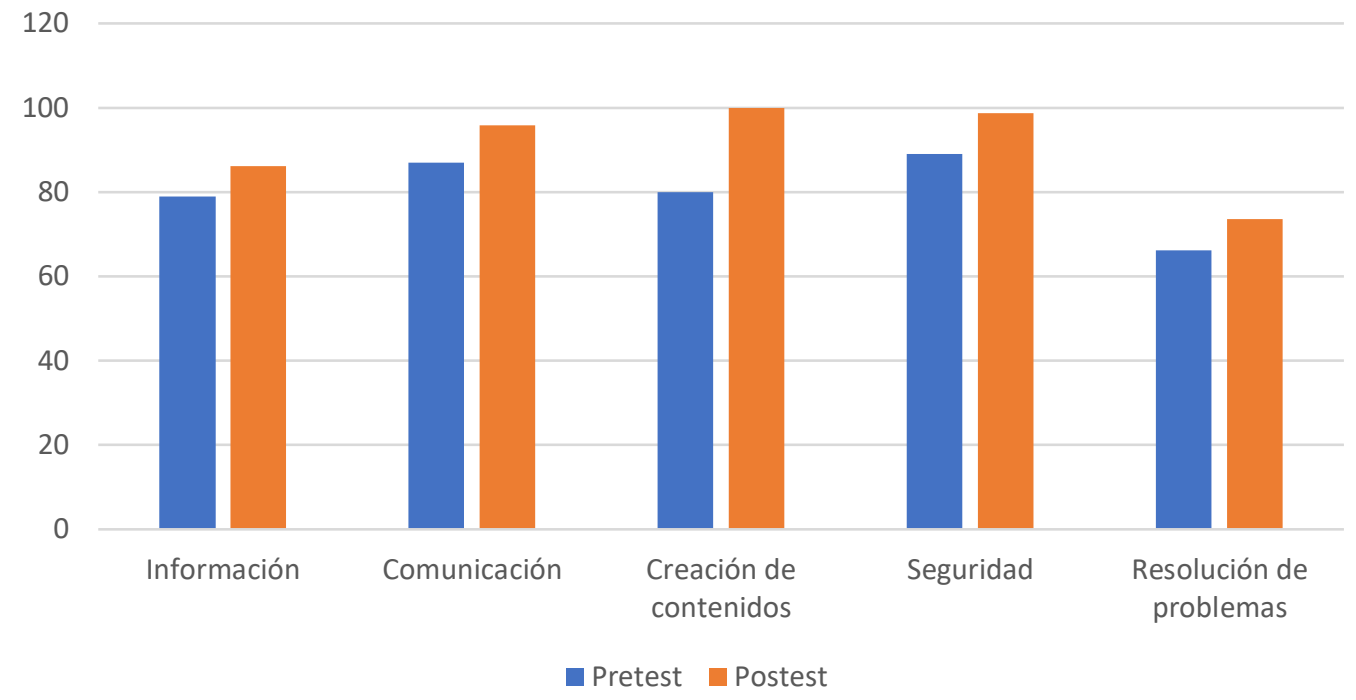

Figura 5. Diferencia en las cinco dimensiones pretest - postest

El Lambda de Wilks fue de $.019, F(5,213)=2166.38, p<.001$, confirmando el valor discriminante de las variables. Después de realizar la prueba de Levene y comprobar la igualdad de varianza, se realizó una ANOVA recogida en la siguiente Tabla 1.

\begin{tabular}{cccc}
\hline \multirow{2}{*}{ Áreas competenciales } & \multicolumn{2}{c}{ Media (Desviación Típica) } & \multirow{2}{*}{ Pretest } \\
& Postest & \\
\hline Información & $78.98(15.43)$ & $86.17(16.01)$ & $11.38^{* * *}$ \\
Comunicación & $87.05(14.44)$ & $95.84(14.96)$ & $19.51^{* * *}$ \\
Creación de contenidos & $79.97(25.67)$ & $100.01(20.89)$ & $40.45^{* * *}$ \\
Seguridad & $88.98(17.08)$ & $98.81(17.44)$ & $17.68^{* * *}$ \\
Resolución de problemas & $66.26(15.37)$ & $73.63(13.56)$ & $14.23^{* * *}$ \\
\hline
\end{tabular}
Nota: ${ }^{* * *} p<.001$

Tabla 1. Media, desviación típica y diferencias estadísticas (F) para el pretest y el postest

\section{DISCUSIÓN Y CONCLUSIONES}

La competencia digital es un elemento clave dentro de la formación de los futuros profesionales de todos los ámbitos, presente en todos los niveles educativos y recogido en las políticas educativas (LOMCE, 2013). Como recoge Area (2010), es necesario formar al alumnado universitario en competencias digitales para que adquiera una serie de habilidades necesarias para su futuro profesional. Se trata de una competencia transversal que puede permitir al alumnado el desarrollo de otras competencias (Ferrari, 2013). Es por ello por lo que se presenta este trabajo, en el que se recoge cómo el hecho de trabajar directamente esta competencia puede ayudar al alumnado a mejorar en la misma.

Los alumnos participantes en este trabajo son alumnos que se encuentran a mitad de sus estudios de Grado, siendo esta la primera asignatura que tienen en la titulación de 
Pedagogía sobre Tecnología Educativa. Como se ha indicado anteriormente, el trabajo se dispuso en una serie de fases: en primer lugar, la autoevaluación por parte del alumnado de su nivel de competencia digital, en segundo lugar el alumnado realizó una serie de actividades para trabajar cada una de las áreas competenciales siguiendo el modelo DigComp y la última fase en la que los estudiantes volvieron a realizar la autoveluación de su nivel de competencia digital.

Al inicio del curso los alumnos mostraron en general un nivel medio de competencia digital en todas las áreas. Sin embargo, destaca el hecho de que, en las áreas de Resolución de Problemas, seguido de Información y de Creación de contenidos, dónde encontramos los valores medios más bajos. Al contrario, en Comunicación y en Seguridad obtienen mejor media, lo cual está en relación con el estudio de Gutiérrez, Román y Sánchez (2018) en el que se determinó que usaban herramientas de red social de forma habitual. El hecho de que el nivel competencial de los alumnos no sea excesivamente alto contrasta con la distinción de "nativos digitales" que realizó Prensky en 2001, siendo en este caso una muestra de estudiantes considerados como nativos. Concuerda, por el contrario, con otros trabajos en los que los estudiantes no mostraron una gran habilidad del uso de estas herramientas para el aprendizaje (Prendes y Román, 2017). También está en relación con otros estudios en los que se muestra como los alumnos llegan a la universidad con un nivel básico de la competencia digital (Gisbert y Esteve, 2011; Gutiérrez y Serrano, 2016).

En cuanto a las diferencias iniciales entre hombres y mujeres, teniendo en cuenta la diferencia de muestra, destaca el hecho de que los varones obtienen medias superiores en todas las áreas competenciales, siendo esta diferencia significativa en las áreas de Información, Seguridad y Resolución de Problemas. Este dato difiere con el estudio de López, González, Aguiar y Artiles (2017) en el que encontraron que las mujeres usaban más buscadores generalistas y bases de datos especializadas que los varones.

Después de finalizar la asignatura se evaluaron los alumnos con el mismo instrumento. En esta ocasión, todas las medias obtenidas son superiores al inicio de curso, siendo igualmente la Resolución de Problemas el área con la media más baja, seguido del área de Información. Es llamativo como en el área de Creación de contenidos la media obtenida pasa a ser la más alta. En las diferencias por sexo, si bien al inicio se encontraron diferencias en el área de Información y Seguridad, ahora ya no existen diferencias significativas en ambas, encontrando solo diferencias significativas en el área de Resolución de problemas.

Pero lo más destacable de este trabajo son las diferencias estadísticas encontradas en las cinco áreas competenciales entre el antes y el después de realizar las tareas. En todos los casos, las diferencias entre el pretest y el postest fueron significativas, destacando el caso del área de Creación de contenidos donde la media aumentó considerablemente. Mientras que, por el contrario, donde los alumnos siguen mostrando más carencia es en la Resolución de Problemas, contrastando con el estudio de Gutiérrez y Serrano (2016) donde los alumnos tuvieron un menor nivel en el área de Seguridad. Este dato seguramente sea debido a que en todas las tareas, aunque su objetivo fuera el trabajo de otra área competencial, creaban distinto contenido que tenían que publicar en su blog de grupo. 
En general, destaca que el trabajo de la competencia digital ayuda a que el alumno sea consciente de su nivel competencial y lo vaya mejorando a partir de la propia práctica relacionada con su futura profesión (Gutiérrez y Serrano, 2016; Prendes, Castañeda y Gutiérrez, 2010). Sin embargo, ese mismo hecho es una limitación de este estudio, ya que son los alumnos los que evaluaban su competencia digital a través de un cuestionario de autopercepción. Sería conveniente realizar algún tipo de prueba de habilidad que permitiera conocer con datos objetivos el antes y después de los alumnos. Además, en el propio estudio no se han tenido en cuenta otras variables que pudieran afectar al desarrollo de este trabajo, como podría ser el conocimiento previo de manejo de las TIC.

Por tanto, sería necesario plantear un estudio en el que se controlaran otra serie de variables y se utilizara otro tipo de evaluación más objetiva para poder determinar con exactitud que los alumnos alcanzan una mejor competencia digital, o realizar un estudio longitudinal.

\section{REFERENCIAS}

Area, M. (2010). ¿Por qué formar en competencias informacionales y digitales en la educación superior? RUSC, Revista de Universidad y Sociedad del Conocimiento, 7(2), 2-5. Recuperado de http://rusc.uoc.edu/rusc/ca/index.php/rusc/article/download/v7n2-area/976-10111-PB.pdf

Cabezas, M.; Casillas, S. y Pinto, A.M. (2014). La percepción de los alumnos de Educación Primaria de la Universidad de Salamanca sobre su competencia digital. EDUTEC, 48, 1-14. doi: 10.21556/edutec.2014.48.114

Carretero, S., Vuorikari, R. y Punie, Y. (2017). DigComp 2.1. The digital Competence Framework for Citizens. With eight proficiency levels and examples of use. Recuperado de Comisión Europea website http://publications.jrc.ec.europa.eu/repository/bitstream/JRC106281/webdigcomp2.1pdf_(online).pdf

Comisión Europea (2006). Competencias clave para el aprendizaje permanente. Recomendación 2006/962/CE del Parlamento Europeo y del Consejo, de 18 de diciembre de 2006, sobre las competencias clave para el aprendizaje permanente [Diario Oficial L 394 de 30.12.2006]

Comisión Europea (2014). Comprender las políticas de la Unión Europea: Agenda Digital para Europa. Recuperado de http://europa.eu/pol/index_es.htm

Comisión Europea (2016). DigCompOrg. Digitally Competent Educational Organisations. Recuperado de https://ec.europa.eu/jrc/en/digcomporg

Davila, S. (2006). Generación Net: Visiones para su Educación. ORBIS, 3, 24-48. Recuperado de http://www.revistaorbis.org.ve/pdf/3/3Art2.pdf 
Ferrari, A., Neza, B. y Punie, Y. (2014). DIGCOMP: A Framework for Developing and Understanding Digital Competence in Europe. eLearning Papers, 38, 3-17. Recuperado de www.openeducationeuropa.eu/en/elearning_papers

Flores-Lueg, C.B. y Roig-Vila, R. (2016). Diseño y validación de una escala de autoevaluación de competencias digitales para estudiantes de Pedagogía. PíxelBit. Revista de Medios y Educación, 48, 209-224. doi: 10.12795/pixelbit.2016.i48.14

Gisbert, M. y Esteve, F. (2011). Digital Leaners: la competencia digital de los estudiantes universitarios. La Cuestión Universitaria, 7, 48-59.

Gisbert, M., Espuny, C., y González, J. (2011). INCOTIC. Una herramienta para la @utoevaluación diagnóstica de la competencia digital en la universidad. Profesorado, revista de currículum y formación de profesorado, 15(1), 76-89.

Gutiérrez Porlán, I. (2014). Perfil del profesor universitario español en torno a las competencias en tecnologías de la información y la comunicación. Pixel-Bit, Revista de Medios y Educación, 44, 51-65. doi: 10.12795/pixelbit.2014.i44.04

Gutiérrez, I. y Serrano, J.L. (2016). Evaluation and development of digital competence in future primary school teachers at the University of Murcia. Journal of New Approaches in Educational Research, 5(1), 51-56. doi: 10.7821/naer.2016.1.152

Gutiérrez, I., Román, M. y Sánchez, M.M. (2018). Estrategias para la comunicación y el trabajo colaborativo en red de los estudiantes universitarios. Revista Comunicar, 54, 91-100. doi: 10.3916/C54-2018-09

INTEF, Instituto Nacional de Tecnologías Educativas y de Formación del Profesorado (2013). Marco común de competencia digital docente. Recuperado de: http://educalab.es/documents/10180/12809/MarcoComunCompeDigiDoceV2.pdf

INTEF, Instituto Nacional de Tecnologías Educativas y de Formación del Profesorado (2017). Organizaciones Educativas Digitalmente Competentes. Recuperado de http://educalab.es/intef/digcomp/digcomporg

INTEF, Instituto Nacional de Tecnologías Educativas y de Formación del Profesorado (2017). Marco Común de Competencia Digital Docente. Recuperado de http://aprende.intef.es/sites/default/files/2018-05/2017_1020_Marco-

Com\%C3\%BAn-de-Competencia-Digital-Docente.pdf

Kampylis, P., Punie, Y. y Devine, J. (2016). Promoción de un aprendizaje eficaz en la era digital. Un marco europeo para organizaciones educativas digitalmente competentes.

Recuperado

de

https://sede.educacion.gob.es/publiventa/d/21199/19/000

Krumsvik, R. (2011). Digital competence in Norwegian teacher education and schools. Högre utbildning, 1(1), 39-51. Recuperado de http://journals.lub.lu.se/index.php/hus/article/view/4578

Ley Orgánica 2/2006, de 3 de mayo, de Educación. Boletín Oficial del Estado, núm. 106, de 4 de mayo de 2006, pp. 17158 a 17207. https://www.boe.es/boe/dias/2006/05/04/pdfs/A17158-17207.pdf 
Ley Orgánica 8/2013, de 9 de diciembre, para la mejora de la calidad educativa. Boletín Oficial del Estado, núm. 295, de 10 de diciembre de 2013, pp. 97858 a 97921. http://www.boe.es/boe/dias/2013/12/10/pdfs/BOE-A-2013-12886.pdf

Marín Trejo, R. (2017). Diseño y evaluación de un instrumento de la competencia digital docente (Tesis Doctoral). Universidad de Islas Baleares.

Monereo, C (2005). Internet, un espacio idóneo para desarrollar las competencias básicas. En Monerreo, C. (Coord.), Internet y competencias básicas: aprender a colaborar, a comunicarse, a participar, a aprender (pp. 5-26). Barcelona: Graó.

OCDE, Organización para la Cooperación y el Desarrollo Económico, (2005). The definition and selection of key competences. Executive Summary. Recuperado de http://www.oecd.org/pisa/35070367.pdf. Traducción al español en http://deseco.ch/bfs/deseco/en/index/03/02.parsys.78532.downloadList.94248.Do wnloadFile.tmp/2005.dscexecutivesummary.sp.pdf

Pavié, A. (2011). Formación docente: hacia una definición del concepto de competencia profesional docente. Revista Electrónica Interuniversitaria de Formación del Profesorado, 14(1), 67-80. Recuperado de http://www.aufop.com/aufop/uploaded_files/articulos/1301587967.pdf

Petterson, F. (2017). On the issues of digital competence in educational contexts: a review of literature. Education and information technologies, 23, 1005-1021. doi: 10.1007/s10639-017-9649-3

Prendes Espinosa, M.P. y Román García, M.M. (2017). Entornos Personales de Aprendizaje. Una visión actual de cómo aprender con tecnologías. Barcelona: Octaedro.

Prendes, M.P., Castañeda, L. y Gutiérrez, I. (2010). Competencia para el uso de TIC de los futuros maestros. Revista Comunicar, 35, 175-182. doi: 10.3916/C35-201003-11

Roig Vila, R. y Pascual Luna, A.M. (2012). Las competencias digitales de los futuros docentes. Un análisis con estudiantes de Magisterio de Educación Infantil de la Universidad de Alicante. @tic, Revista d'Innovació Educativa, 9, 53-60. Recuperado de http://ojs.uv.es/index.php/attic/article/view/1958

Roig Vila, R., Rodríguez-Cano, C., Flores, C., Álvarez, J.D., Blasco, J.E., Grau, S. y Tortosa, M.T. (2012). Evaluación de las Competencias Digitales del alumnado en el Espacio Europeo de Educación Superior. En M.T. Tortosa, J.D. Álvarez y N. Pellín (Coord.), X Jornadas de Redes de Investigación en Docencia Universitaria: la participación y el compromiso de la comunidad universitaria (pp. 781-795). Recuperado de https://web.ua.es/es/ice/jornadas-redes2012/documentos/posters/243944.pdf

UNESCO (2011). UNESCO ICT Competency Framework for Teachers. Recuperado de http://unesdoc.unesco.org/images/0021/002134/213475e.pdf 
White D. y Le Cornu, A. (2011). Visitors and Residents: a new typology for online engagement. First Monday, 16(9). Recuperado de http://www.uic.edu/htbin/cgiwrap/bin/ojs/index.php/fm/article/viewArticle/3171/30 $\underline{49}$

\section{Para referenciar este artículo:}

González Calatayud, V., Román García, M., \& Prendes Espinosa, M.P. (2018). Formación en competencias digitales para estudiantes universitarios basada en el modelo DigComp. Edutec. Revista Electrónica de Tecnología Educativa, 65, 1-15. doi:https://doi.org/10.21556/edutec.2018.65.1119 